\title{
Síndrome de embolismo graso postraumático: reporte de un caso
}

Abel Arroyo-Sánchez ${ }^{1,3}$, Rosa Aguirre-Mejía ${ }^{2,3}$

RESUMEN

El síndrome de embolismo graso (SEG) post traumático es una complicación potencialmente letal y poco sospechada. Se presenta un caso de SEG diagnosticado clínicamente y confirmado por los criterios diagnósticos vigentes; cuyas características epidemiológicas, factores de riesgo, cuadro clínico y evolución favorable después del tratamiento recibido, fueron de acuerdo a lo publicado. Se realiza una revisión de la literatura y se proponen medidas para disminuir el riesgo de su presentación y mejorar el diagnóstico oportuno.

Palabras clave: Embolia grasa; traumatismo múltiple; fracturas del fémur (Fuente: DeCS BIREME).

\section{Post-traumatic fat embolism syndrome: a case report}

\section{ABSTRACT}

Post-traumatic fat embolism syndrome (FES) is a potentially lethal and poorly suspected complication. We present a case of FES diagnosed clinically and confirmed by current diagnostic criteria. The epidemiological characteristics, risk factors, clinical features and favorable evolution after treatment were as reported. We review the literature and propose measures to reduce the risk of its presentation and improve early diagnosis.

Keywords: Fat embolism; multiple trauma; femoral fractures (Source: MeSH NLM).

1. Hospital Víctor Lazarte Echegaray, Servicio Cuidados Intensivos. Trujillo, Perú.

2. Hospital Víctor Lazarte Echegaray, Servicio Diagnóstico por Imágenes. Trujillo, Perú.

3. Universidad Privada Antenor Orrego, Facultad de Medicina Humana. Trujillo, Perú. 


\section{INTRODUCCIÓN}

La muerte y discapacidad generadas por los accidentes vehiculares son un problema de salud pública en los países en vías de desarrollo y a nivel mundial, sobre todo entre la segunda y cuarta décadas de la vida ${ }^{(1)}$. La mayoría de los pacientes con trauma severos presentan lesiones y complicaciones, que son evidentes de manera precoz, lo que permite un diagnóstico y tratamiento oportunos. Pero, algunos pacientes pueden presentar complicaciones potencialmente mortales dentro de las 12 - 72 hrs después de haber ocurrido el trauma, con una identificación difícil o tardía si no se las tiene en consideración, una de ellas es el síndrome de embolia grasa (SEG).

\section{CASO CLÍNICO}

Hombre de 30 años, etnia mestiza, soltero y sin antecedente de enfermedad previa, alergias o consumo de drogas; quien conduciendo su vehículo sufre un impacto frontal en un accidente automovilístico 6 horas antes de su ingreso a la emergencia, llega despierto, orientado, cooperador, refiriendo dolor en pelvis, muslos y hemitórax izquierdo.

Al examen físico con presión arterial: 103/65 mmHg; temperatura axilar: $36,8{ }^{\circ} \mathrm{C}$; frecuencia respiratoria 20 rpm; frecuencia cardiaca: 98 lpm; saturación: 98 \% (FiO2: $0,21)$. Se evidencia equimosis en cabeza y múltiples escoriaciones en el cuerpo. Escala de Coma de Glasgow (ECG) de 15 puntos; ruidos cardiacos regulares, rítmicos sin ruidos agregados; campos pulmonares ventilados sin ruidos anormales, con dolor a la presión en parrilla costal izquierda; abdomen con ruidos hidroaéreos disminuidos, a la palpación blando y depresible, sin signos peritoneales; genitales externos sin lesiones pero se evidencia hematuria macroscópica a través del meato urinario, con desarrollo progresivo de globo vesical que amerita la realización de una talla vesical de emergencia; edema y asimetría de ambos muslos con limitación del movimiento y sin exposición ósea, con pulsos periféricos presentes y sensibilidad conservada.

Se le realiza analítica obteniendo: hemoglobina 11,6 $\mathrm{gr} / \mathrm{dL}$; leucocitos $15000 / \mathrm{mm} 3$ (12\% abastonados); plaquetas $212000 / \mathrm{mm} 3$; glucemia $142 \mathrm{mg} / \mathrm{dL}$; urea $35 \mathrm{mg} / \mathrm{dL}$; creatinina $0,8 \mathrm{mg} / \mathrm{dL}$; gases arteriales sin oxígeno suplementario, con pH 7,34; pO2 $79 \mathrm{mmHg}$ ( $\mathrm{Pa} /$ FiO2: 376; gradiente A-a: 23,2 mmHg); pCO2 $38 \mathrm{mmHg}$; bicarbonato $20 \mathrm{mEq} / \mathrm{L}$; BE $-5 \mathrm{mEq} / \mathrm{L}$ y saturación $95 \%$. Examen de orina con hemoglobina $50 \mathrm{hmt} / \mathrm{uL}$; nitritos negativos, hematuria 15 /campo AP; leucocitos $1 /$ campo AP.

Las tomografías computarizadas de ingreso concluyeron: No lesión parenquimal cerebral ni ósea craneal; fractura de la $8^{a}$ y $9^{a}$ costillas izquierdas y campos pulmonares sin compromiso pleuroparenquimal; no lesiones intrabdominales ni líquido libre; fractura pélvica y femoral bilateral (Figuras 1 y 2).

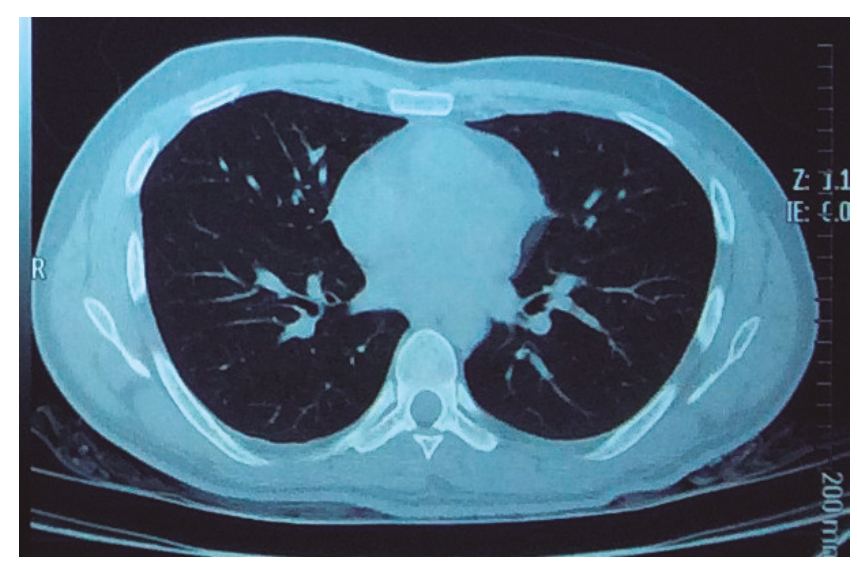

Figura 1. Tomografía de pulmones de ingreso sin lesiones pleuroparenquimales 


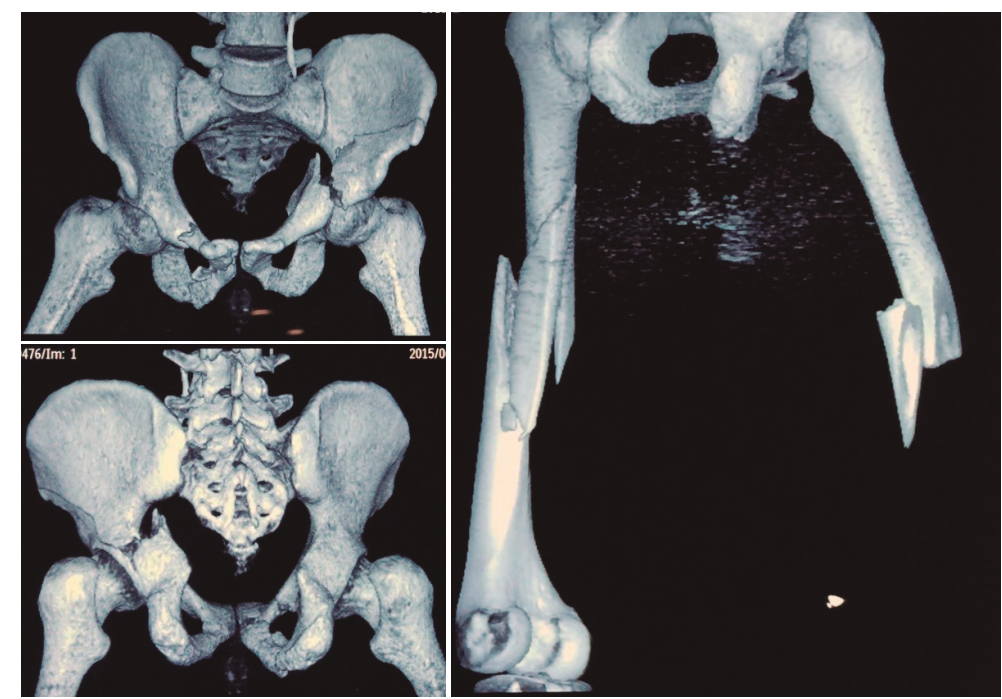

Figura 2. Reconstrucción tomográfica de pelvis y fémur bilateral mostrando: doble fractura del hueso iliaco izquierdo y rama isquio e ilio púbica derecha, y fractura femoral bilateral

Ingresa a Cuidados Intensivos para tratamiento y monitoreo con un Índice de Severidad del Trauma (ISS) de 29 puntos y un Trauma Score Revisado (RTS) de 7,84 puntos. A las 25 horas de su ingreso al hospital ( 31 horas de ocurrido el accidente), presenta súbitamente presión arterial 145/96 mmHg; taquicardia supraventricular hasta 151 lpm; desaturación 82 \% (FiO2 0,21); afebril $36,3^{\circ} \mathrm{C}$; hiperventilación intermitente; dolor y leve agitación psicomotriz. Se aplica analgésico, sedación suave y oxígeno suplementario con lo cual mejoran sus signos vitales: presión arterial 100/60 mmHg; frecuencia cardiaca 100 lpm; temperatura $36,5^{\circ} \mathrm{C}$; y saturación 100 \% (FiO2 0,4 por cánula nasal).

A las $29 \mathrm{~h}$ de su ingreso (35 h del accidente) presenta súbitamente hipotensión arterial $80 / 50 \mathrm{mmHg}$, taquicardia supraventricular 160 lpm, desaturación $70 \%$ (FiO2 0,4), y polipnea con signos de dificultad respiratoria. Se coloca tubo orotraqueal, sedación/ analgesia, soporte de ventilación mecánica con FiO2 1,0 , fluidoterapia y vasopresor; se evidencian petequias en tórax anterior, cuello y axilar (Figura 3).

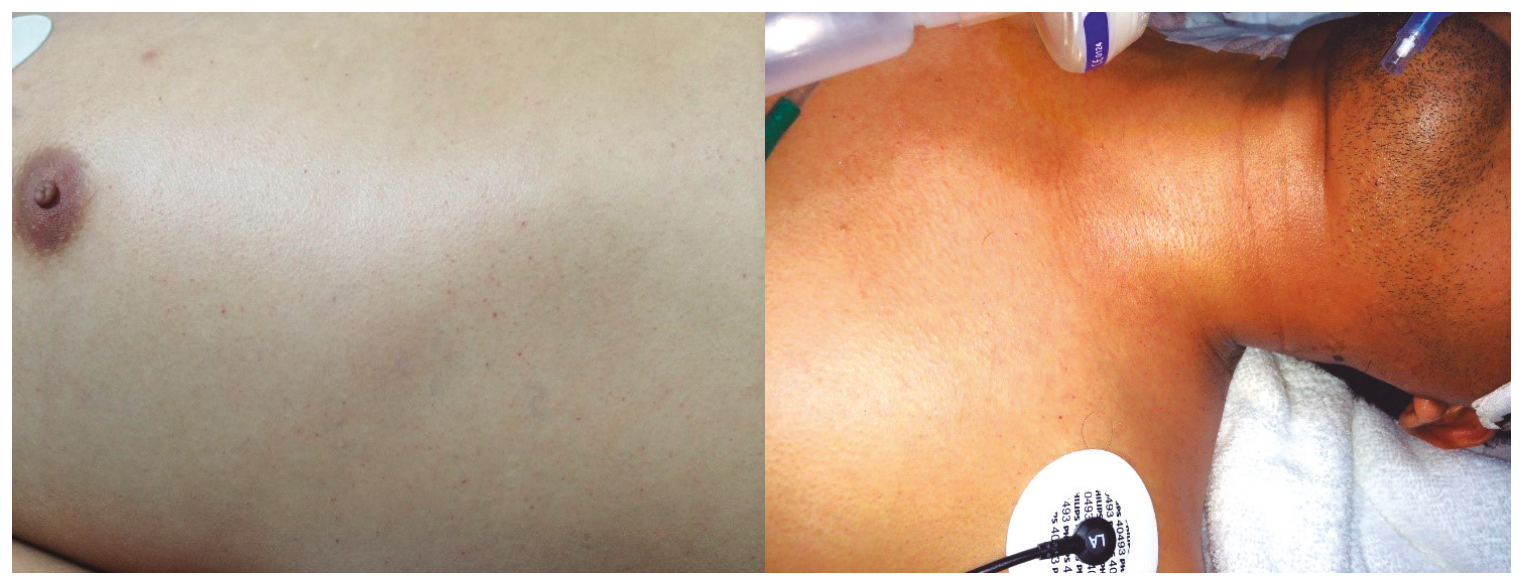

Figura 3. Lesiones petequiales en tórax antero bilateral y base del cuello

Ante la sospecha clínica de SEG se actualizan los análisis de laboratorio y se evidencia hemoglobina 9,7 gr/dL; leucocitos $10300 / \mathrm{mm} 3$ (3 \% abastonados); plaquetas $68000 / \mathrm{mm} 3$; urea 33 mg/dL; creatinina 0,7 mg/dL; sodio 144 $\mathrm{mEq} / \mathrm{L}$; potasio 3,1 mEq/L; cloro $111 \mathrm{mEq} / \mathrm{L}$; gases arteriales en ventilación mecánica con FiO2 0,5: pH 7,30; pO2 93 mmHg (Pa/Fi: 186; gradiente A-a: 213,5 mmHg); pCO2 40 mmHg; bicarbonato $20 \mathrm{mEq} / \mathrm{L}$; BE -7 mE/L y saturación $96 \%$. Velocidad de sedimentación globular de $22 \mathrm{~mm} / \mathrm{h}(\mathrm{V} . \mathrm{N}$. hasta $15 \mathrm{~mm} / \mathrm{h})$. La radiografía de tórax portátil de control muestra un infiltrado alveolar bibasal (Figura 4). 


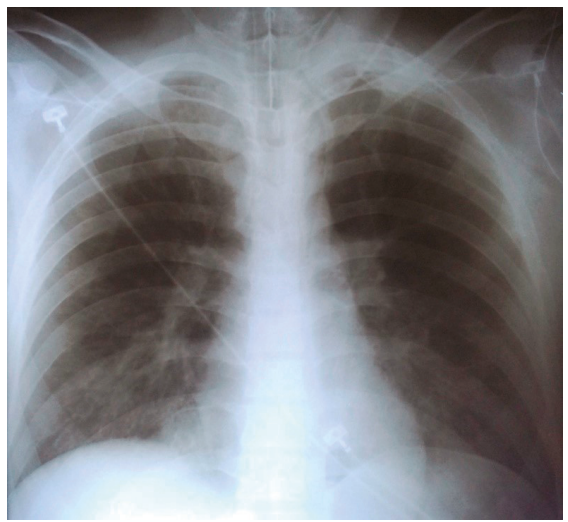

Figura 4. Radiografía anteroposterior del tórax con infiltrado alveolar basal bilateral

Se continúa con soporte avanzado de la vida incluyendo hemotransfusión. Oftalmología no encuentra evidencia de embolia en el fondo de ojo. Se realiza una TC de cerebro que evidencia foco hiperdenso de aspecto hemorrágico en la sustancia blanca a nivel de los centros semiovales izquierdos (Figura 5A); y una resonancia magnética nuclear de cerebro sin contraste, encontrando signos de lesión axonal difusa con compromiso edematoso a foco múltiple en hemisferios cerebrales y cuerpo calloso, además focos hemorrágicos menores de $5 \mathrm{~mm}$ en centros semiovales y periventricular (Figuras 5B a 5F).

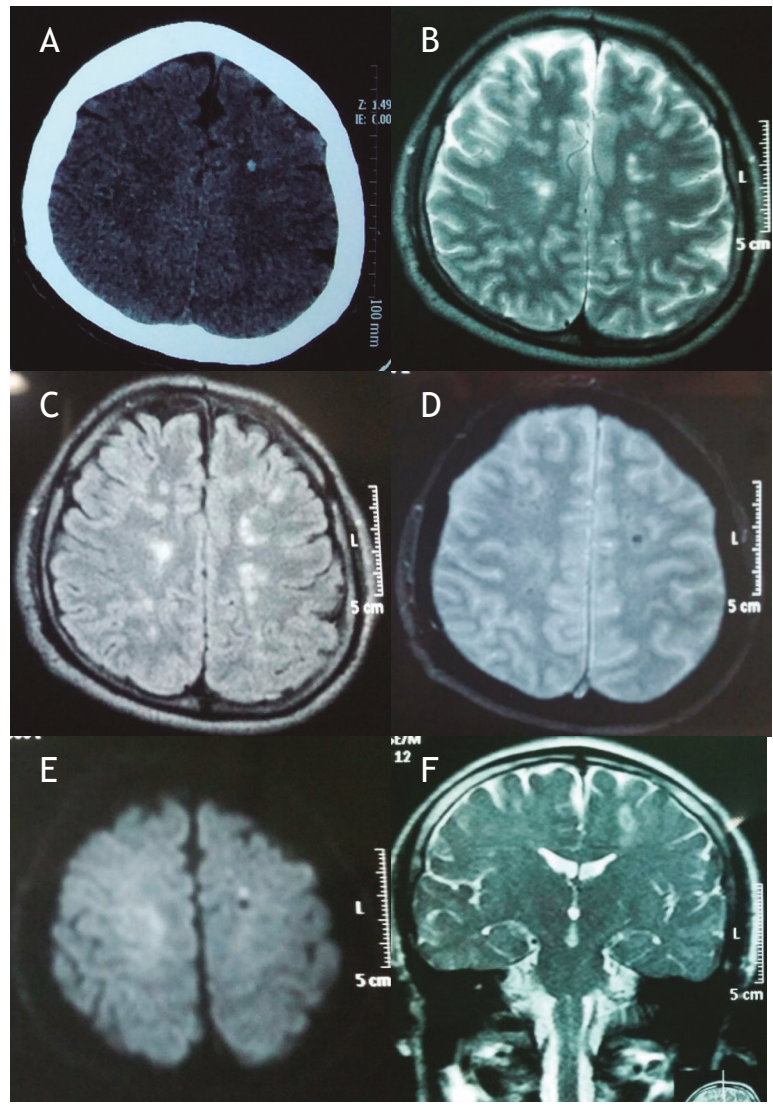

Figura 5. TC cerebral con foco hiperdenso de aspecto hemorrágico en la sustancia blanca a nivel de los centros semiovales izquierdos al sexto día del trauma (A). RMN axial y coronal cerebral al octavo día del trauma evidenciando en T2 áreas hiperintensas en los centros semiovales en relación a edema (B y F, respectivamente); áreas de hiperseñal en la sustancia blanca y los centros semiovales en FLAIR (C); pérdida de la señal focal en centro semioval izquierdo con supresión grasa (D); áreas de restricción en relación a zonas de infartos en la difusión (E). 
A las $48 \mathrm{~h}$ de haber iniciado el SEG, el paciente tolera la disminución progresiva del vasopresor, la $\mathrm{FiO2}$ y la sedoanalgesia. A las $96 \mathrm{~h}$ del SEG, el paciente es retirado del ventilador mecánico y extubado con ECG 10 puntos (respuesta ocular: 3/ verbal: 1/motora: 6).

Se realiza la reducción cruenta de las fracturas femorales al $7^{\circ}$ día de haber ingresado al hospital y la de cadera al $12^{\circ}$ día. Fue dado de alta de Cuidados Intensivos el 9no día con una ECG 15 puntos, orientado en espacio y persona, y parcialmente orientado en tiempo.

\section{DISCUSIÓN}

El embolismo graso consiste en la presencia de glóbulos de grasa en el torrente sanguíneo generalmente asociado a un traumatismo con lesión ósea, pero sin relevancia clínica, de acuerdo a lo identificado en los pulmones de pacientes traumatizados y fracturados que fallecieron de causas diferentes al trauma per se. En cambio, el síndrome de embolismo graso (SEG) es una complicación potencialmente mortal que puede pasar desapercibida y se caracteriza además del embolismo graso por confusión mental, dificultad respiratoria y lesiones petequiales en piel y mucosas ${ }^{(2)}$.

Los traumatismos y la cirugía de trauma y ortopedia se asocian frecuentemente al SEG, aunque la cirugía estética también puede precederla ${ }^{(3,4)}$. La frecuencia de su identificación dependerá de los criterios usados para su diagnóstico y la población estudiada; Gurd y Wilson ${ }^{(2)}$, lo identificaron en el $19 \%$ de los pacientes con trauma severo en el Royal Victoria Hospital en un periodo de 4 años; mientras que Tsai et al ${ }^{(5)}$, encontraron en Taiwán una incidencia $<5 \%$ estudiando pacientes solo con fracturas de huesos largos en un periodo de 11 años. La mayoría de autores coincide en que el SEG es más frecuente en el trauma, entre los 10 a 40 años de edad, varones más que mujeres, múltiples fracturas de huesos largos y cadera, y en fracturas no expuestas ${ }^{(2-4,6-8)}$. El caso que presentamos tenía los factores epidemiológicos asociados al desarrollo de SEG: masculino, 30 años de edad, fractura no expuesta bifemoral, pelvis y costales.

El mecanismo fisiopatológico del SEG aún no está totalmente dilucidado, pero se plantean varias teorías; la de la intravasación, donde la grasa ingresa al torrente sanguíneo venoso después del trauma, o una cirugía ortopédica que aumente la presión intramedular durante una fijación; la obstrucción mecánica, del embolo graso que disminuye el flujo sanguíneo cardiaco de derecha a izquierda; la bioquímica, mediante la cual la lipasa sérica se eleva hidrolizando la grasa circulante en triacilglicerol y ácidos grasos libres, teniendo estos últimos una alta toxicidad para las unidades alveolo capilares; y la teoría de la coagulación, que siguiendo a la lesión del vaso medular, después del trauma o una intervención quirúrgica, genera micro y macro émbolos que potencian el fenómeno obstructivo circulatorio de la grasa generando, adicionalmente a lo antes referido, una respuesta infamatoria cuya magnitud dependerá del volumen total de los émbolos y las condiciones basales previas del paciente ${ }^{(2-4,6,9)}$. Algunos glóbulos grasos pasan el filtro natural pulmonar por el tamaño de sus partículas o por defectos en los tabiques interauricular 0 interventricular volviéndose émbolos sistémicos ${ }^{(3,10)}$.

En todos los casos de SEG, asociado al trauma, existe un periodo de latencia que varía en promedio de 12 . 72 horas después del evento inicial y excepcionalmente puede ser menor de 12 horas o llegar hasta las 2 semanas $(2,3,4,6,7,8,11-14)$; nuestro paciente inició la triada clínica de sospecha a las 25 horas de ingreso hospitalario (31 horas del evento) y se completó la misma a las 29 horas (35 horas del evento).

La presentación del SEG puede ser leve, severa y fatal; dentro de las manifestaciones clínicas iniciales la dificultad respiratoria, asociada a hipoxemia, es la más frecuente (en 75-95\% de los casos) y puede ser documentada con el análisis de gases arteriales y la aparición de infiltrados pulmonares nuevos en los estudios de imágenes ${ }^{(2,3,4,6-9)}$. El compromiso neurológico es la segunda manifestación frecuente, puede presentarse desde la confusión hasta el coma, y la sospecha aumentará si el paciente estaba previamente con una escala de coma de Glasgow de 15 puntos, debiendo corroborarse una lesión estructural nueva en los estudios de imágenes, sobretodo la resonancia magnética nuclear (RMN) y cuyos hallazgos varían de acuerdo al tiempo transcurrido después del trauma (15); en la mayoría de los casos el compromiso neurológico es temporal y reversible ${ }^{(2,3,4,6-9)}$. El rash petequial, es la tercera manifestación en frecuencia, afecta principalmente en la región peri axilar, base del cuello y menos frecuente en conjuntiva y mucosas ${ }^{(2,3,4,6-9,11)}$; en nuestro caso se hicieron evidentes en tórax anterior, cuello y axilas.

Otros hallazgos clínicos y de laboratorio también importantes y que ayudan al diagnóstico son la fiebre, taquicardia, oliguria o anuria, ictericia, retinopatía grasa o petequial, anemia y/o trombocitopenia agudas, elevación de la velocidad de sedimentación globular (VSG), glóbulos grasos en sangre u orina ${ }^{(2,3,4,6,-9)}$.

Ala fecha no existen criterios diagnósticos estandarizados y validados prospectivamente, por lo tanto, al no haber un estándar de oro para el diagnóstico debemos recurrir a los antecedentes, cuadro clínico, exámenes de apoyo diagnóstico y a los criterios propuestos por investigadores como Gurd y Wilson ${ }^{(4)}$, Lindeque et al (3) o Schonfeld et al (9). En las tablas se describen los criterios diagnósticos para cada autor y los encontrados en nuestro paciente. (Tablas 1,2 y 3 ) 
Tabla 1. Criterios diagnósticos de síndrome de embolia grasa de Gurd y Wilson

\begin{tabular}{|c|c|c|}
\hline & Criterios & Caso \\
\hline \multirow[t]{7}{*}{ Mayores } & Rash petequial & Presente \\
\hline & $\begin{array}{l}\text { Síntomas respiratorios con } \\
\text { cambios radiográficos }\end{array}$ & Presente \\
\hline & $\begin{array}{l}\text { Signos neurológicos no } \\
\text { relacionados al trauma u } \\
\text { otra condición. }\end{array}$ & Presente \\
\hline & Taquicardia & Presente \\
\hline & Pirexia & --- \\
\hline & $\begin{array}{l}\text { Retinopatía (grasa o } \\
\text { petequial) }\end{array}$ & -- \\
\hline & $\begin{array}{l}\text { Nefropatía (oliguria, anuria o } \\
\text { lipiduria) }\end{array}$ & --- \\
\hline \multirow{4}{*}{ Menores } & Trombocitopenia aguda & Presente \\
\hline & Anemia aguda & Presente \\
\hline & $\begin{array}{l}\text { Elevada velocidad de } \\
\text { sedimentación globular }\end{array}$ & Presente \\
\hline & Glóbulos grasos en esputo & --- \\
\hline
\end{tabular}

Diagnóstico de SEG: Un criterio mayor y cuatro menores.

Tabla 2. Criterios diagnósticos de síndrome de embolia grasa de Lindeque et al

$\begin{array}{ll}\text { Criterios } & \text { Caso } \\ \text { Presión arterial de Oxígeno }<60 \mathrm{mmHg}(\mathrm{FiO} 2 \text { 0.21) o PaO2/FiO2 }<286 & \text { Presente* }^{*} \\ \text { Presión arterial de } \mathrm{CO} 2>55 \mathrm{mmHg} \text { o pH }<7.30 & -- \\ \text { Frecuencia respiratoria sostenida }>35 \mathrm{rpm} & -- \\ \text { Incremento del trabajo respiratorio. } & \text { Presente }\end{array}$

Diagnóstico de SEG: Fractura de hueso largo con uno o más criterios.

*PaO2/FiO2 (Presión arterial O2/Fracción inspiratoria 02) del paciente: 186.

Tabla 3. Criterios diagnósticos de síndrome de embolia grasa de Schonfeld et al

$\begin{array}{lll}\text { Criterios } & \text { Caso } & \\ \text { Petequias difusas } & 5 & \text { Presente } \\ \text { Infiltrados alveolares } & 4 & \text { Presente } \\ \text { Hipoxemia }(<70 \mathrm{mmHg}) \text { o } \mathrm{PaO} 2 / \mathrm{FiO} 2<333 & 3 & \text { Presente* } \\ \text { Confusión } & 1 & \text { Presente } \\ \text { Fiebre }>38^{\circ} \mathrm{C} & 1 & \text {--- } \\ \text { Frecuencia cardiaca }>120 \text { latidos por minuto } & 1 & \text { Presente }\end{array}$

Diagnóstico de SEG: Cinco o más puntos en los primeros 3 días de hospitalización. *PaO2/FiO2 (Presión arterial 02/Fracción inspiratoria 02) del paciente: 186. 
Actualmente no existe un tratamiento específico para el SEG, pero el tratamiento aceptado y recomendado es el de soporte vital avanzado en cuidados intensivos y que dependerá de la severidad del compromiso de órganos: resucitación con fluidos, vasopresores, oxigenoterapia, soporte ventilatorio, protección de vía aérea, tromboprofilaxis, terapia de reemplazo renal y membrana extracorpórea en los casos más severos y refractarios al manejo estandarizado. El uso de heparinas a dosis terapéutica, aspirina, N-Acetilcisteina y glucocorticoides no ha demostrado mejorar los resultados de forma contundente y pueden aumentar el riesgo de otras complicaciones ${ }^{(3-4,6-9)}$. La mortalidad por SEG es del 5-15 $\%$ en general, pero puede aumentar hasta el $36 \%$ en los pacientes que requieren ventilación mecánica ${ }^{(3,6,9,13)}$.

Se han descrito medidas que pueden ayudar a prevenir el desarrollo del SEG en los pacientes en riesgo, la estabilización y fijación de las fracturas de huesos largos en el trauma múltiple dentro de las primeras 24 horas, realizar fijación externa antes que intramedular en los casos que se pueda, vaciamiento medular previo ${ }^{(3,6,9)}$. El uso de albúmina como fluido de resucitación y los glucocorticoides profilácticos en algunos estudios han reportado disminución de la morbilidad sin afectar la mortalidad de los casos de SEG, pero requieren estudios prospectivos que validen sus resultados antes de aceptarlos como práctica rutinaria ${ }^{(6,7,8,12)}$.

En conclusión, es importante la identificación precoz de los pacientes con riesgo de presentar SEG (adultos jóvenes, víctimas de trauma con fracturas no expuestas múltiples y de huesos largos) para su prevención y atención oportuna, en caso de desarrollarse, lo que ayudará a disminuir los años de vida saludable perdidos y los costos de la atención sanitaria.

\section{REFERENCIAS BIBLIOGRÁFICAS}

1. Organización Mundial de la Salud [Internet]. Nota descriptiva. Lesiones causadas por el tránsito. 2016. Citado el 06 de diciembre del 2016. Disponible en: http://www.who.int/ mediacentre/factsheets/fs358/es/.

2. Gurd AR, Wilson R. The fat embolism syndrome. Bone \& Joint Journal. 1974;56(3):408-16. Disponible en: https://www. ncbi.nlm.nih.gov/pubmed/4547466

3. Santos LE, Gotes J. Embolia grasa: un síndrome clínico complejo. Rev Inst Nal Enf Resp Mex 2005; 18(3): 230-239. Disponible en: http://scielo.unam.mx/scielo.php?pid=S018775852005000300011\&script=sci_arttext

4. Kosova E, Bergmark B, Piazza G. Fat embolism syndrome. Circulation. 2015;131(3):317-20. Disponible en: http:// europepmc.org/abstract/med/25601951

5. Tsai IT, Hsu CJ, Chen YH, Fong YC, Hsu HC, Tsai CH. Fat embolism syndrome in long bone fracture - clinical experience in a tertiary referral center in Taiwan. J Chin Med Assoc 2010; 73(8): 407-410. Disponible en: http://www.sciencedirect. com/science/article/pii/S1726490110700885

6. Saigal R, Mittal M, Kansal A, Singh Y, Kolar P, Jain S. Fat embolism syndrome. JAPI. 2008;56.Disponible en: https:// www.ncbi.nlm.nih.gov/pubmed/18702388

7. Campo-López C, Flors-Villaverde P, Calabuig-Alborch JR. Síndrome de embolia grasa tras fractura ósea. Rev Clín Esp 2012; 212(10): 482-487. Disponible en: http://www.
sciencedirect.com/science/article/pii/S0014256512003062

8. Berdai AM, Shimi A, Khatouf M. Le síndrome d'embolie graisseuse post traumatique. Pan Afr Med J 2014; 17: 83. Disponible en: https://www.ncbi.nlm.nih.gov/pmc/articles/ PMC4247737/

9. Kwiatt ME, Seamon MJ. Fat embolism syndrome. International journal of critical illness and injury science. 2013;3(1):64.Disponible en: https://www.ncbi.nlm.nih.gov/ pubmed/23724388

10. De Freitas Vellutini D, Ishioka S, de Morais Jr LC. Embolia gordurosa cerebral pós-traumática Cerebral post-traumatic fat embolismo; report of two cases. Arquivos de NeuroPsiquiatria. 1971;29(4):458-62.

11. Calvo JM, Bureo P, Ramos JL. Síndrome de embolismo graso: presentación de un caso y revisión de la literatura. An Med Interna. 2001; 18(11): 62-63. Disponible en: $\quad$ http: //scielo.isciii.es/scielo.php?script=sci_ arttext\&pid=S0212-71992001001100019

12. Bajuri MY, Johan RR, Shukur H. Two variants of fat embolism syndrome evolving in a young patient with multiple fractures. BMJ Case Rep 2013; 2013: bcr2013008631. Disponible en: http:// casereports.bmj.com/content/2013/bcr-2013-008631.long

13. Telford L, Harris J. A traumatic case of fat embolism. BMJ case reports. 2013;2013:bcr2013200142. Disponible en: https:// www.ncbi.nlm.nih.gov/pmc/articles/PMC3736448/

14. Bethany R, Reynolds HN, Bodanapally UK, Dreizin D. Cerebral fat embolism syndrome: diagnostic state of the art: with and without intra-medullary fixation, with and without long bone fractures. Int J Crit Care Emerg Med. 2014; 1.1. Disponible en: https://clinmedjournals.org/articles/ijccem/ international-journal-of-critical-care-and-emergencymedicine-ijccem-1-004.pdf

15. Kuo K-H, Pan Y-J, Lai Y-J, Cheung W-K, Chang F-C, Jarosz J. Dynamic MR imaging patterns of cerebral fat embolism: a systematic review with illustrative cases. American Journal of Neuroradiology. 2014;35(6):1052-7.Disponible en: http:// www.ajnr.org/content/35/6/1052.short

Fuentes de financiamiento:

Este artículo ha sido financiado por los autores.

Conflictos de interés:

Los autores declaran no tener ningún conflicto de interés.

\section{Correspondencia:}

Abel Arroyo Sánchez

Dirección: Jr. Grau 374 Cercado. Trujillo, Perú.

Teléfono: +51947842030

Correo electrónico: abelsalvador@yahoo.com

Recibido: 12 de junio de 2017
Evaluado: 14 de junio de 2017
Aprobado: 28 de agosto de 2017

(c) La revista. Publicado por Universidad de San Martín de Porres, Perú. (c) bajo términos de Licencia Creative Commons Atribución 4.0 Internacional. (http://creativecommons.org/licenses/by/4.0/)

ORCID iDs

Abel Arroyo Sánchez Rosa Aguirre Mejía

https: / / orcid.org/0000-0001-6022-6894 https: / / orcid.org/0000-0002-2283-1935 